\title{
REDESCRIPTION DE L'AMPHIPODE HYPOGÉ NIPHARGUS PUTEANUS (KOCH IN PANZER, 1836), BASÉE SUR DU MATÉRIEL TOPOTYPIQUE
}

\author{
par \\ JAN H. STOCK \\ Institut de Zoologie taxonomique, Université d'Amsterdam, Pays-Bas
}

\begin{abstract}
Niphargus puteanus (Koch in Panzer, 1836) is redescribed, in accordance with the criteria formulated by the "First International Colloquium on the Genus Niphargus", at Verona (1969). The redescription is based on material collected near the type-locality, Regensburg (German Federal Republic). A neotype, and a restricted type-locality, are indicated.
\end{abstract}

\section{INTRODUCTION}

Pendant le „Ier Colloque International sur le genre Niphargus" de Vérone en 1969, un certain nombre de décisions furent prises (voir Ruffo, 1972: viii-ix). La première résolution porta sur la nécessité de refondre la description d'anciennes espèces mal connues ou douteuses, de préférence sur la base de matériel-type primitif ou d'après des topotypes. Sur la liste des espèces entrant en considération pour une nouvelle description, figure en premier lieu Niphargus puteanus; le colloque désigna le docteur M. Straškraba, de Prague, spécialiste dans ce domaine, d'accomplir cette tâche.

Toutefois, pendant le „IIème Colloque International sur les genres Niphargus et Gammarus" de Lyon en 1973, il fut communiqué que, par suite de circonstances indépendantes de sa volonté, le docteur Straß̌kraba ne pouvait remplir la tâche qui lui était confiée; le IIème Colloque a alors désigné l'auteur de ces lignes pour entreprendre, „dans des délais assez brefs" (voir Ginet, sous presse, a $\&$ b), ce travail absolument nécessaire pour mieux comprendre les taxa décrits plus tard. Dans les notes qui suivent, la mission reçue du IIème Colloque est accomplie.

\section{LA DESCRIPTION DE KOCH ET LA STA- TION-TYPE}

Ruffo (1972: viii) cite l'espèce dont la description est à refaire, comme étant „Niphargus puteanus C. L. Koch 1835". L'étude des travaux de Koch a cependant démontré que la description originale ne date pas de 1835 , et, en même temps, que Koch n'a pas décrit son espèce comme étant un Niphargus (ainsi que le fait supposer la citation de Ruffo, sans parenthèses pour isoler le nom de l'auteur), mais comme un Gammarus. De l'ouvrage de Koch, deux éditions ont été publiées, en fascicules. La plus ancienne description se trouve dans le „Faunae Insectorum Germanicae Initia" de Panzer, où „GAMMARUS puteanus Koch”, accompagné d'un dessin (138.2) en couleurs, figure sous le numéro 2 dans le fascicule 138 . Ce fascicule a paru en 1836. La description et l'illustration ont été identiquement réimprimées dans le fascicule $5 \mathrm{du}$,Deutschlands Crustaceen, Myriapoden und Arachniden" de Koch. D'après les investigations de Sherborn (1923), la date de publication de ce fascicule de l'ouvrage de Koch est 1837. Il semble donc justifié d'appeler dorénavant Niphargus puteanus (Koch in Panzer, 1836) l'espèce en question.

Dans un fascicule ultérieur de l'ouvrage de Panzer (186.22), on retrouve Gammarus puteanus, cette fois encore accompagné d'une illustration en couleurs. Selon Schellenberg (1933b:411), les deux espèces désignées comme étant des puteanus, c'est-à-dire les numéros 138.2 et 186.22 dans l'ouvrage de Panzer, ne sont pas identiques. Le numéro 138.2 provient de „Schöpf- und Ziehbrun- 
nen. Bei Regensburg nicht selten" (= puits où l'on puise à la main et puits à poulie. Ne sont pas rares près de Ratisbonne); le numéro 186.22 a été trouvé dans les fontaines (ou puits) de la ville de Deux-Ponts (,,in den Brunnen der Stadt Zweibrücken"). Plus tôt, en 1890, Wrzesniowski avait déjà conclu que les formes de Ratisbonne et de Deux-Ponts ne pouvaient appartenir à la même espèce; c'est pourquoi il rebaptisa la forme ratisbonnienne, l'appelant Niphargus ratisbonensis, une action qui, évidemment, n'est pas justifiée, la forme ratisbonnienne étant la plus ancienne qui ait été décrite. Schellenberg (1933b: 412) soupçonne que la forme trouvée à Deux-Ponts est apparentée ou identique à Niphargus aquilex Schiödte, 1855. et je suis disposé à partager cette opinion.

Les premières mentions d'un Niphargus rencontré dans l'habitat primitif de Koch, à Ratisbonne, se trouvent in Schellenberg, 1933a. Sauf un portrait de l'habitus de la femelle, cette publication ne donne pas de détails sur la morphologie. Cependant, Schellenberg, se basant sur du matériel en provenance du Hochspessart près d'Altenburg en
Basse-Franconie (Bavière, République Fédérale d'Allemagne), a démontré que Niphargus stadleri S. Karaman, $1932: 185$, est synonyme de $N$. puteanus.

On trouvera plus de détails morphologiques dans les publications de S. Karaman, 1932 (sub nomen $N$. stadleri), Schellenberg, 1933b (fig. 4) et Schellenberg, 1932 (sub nomen $N$. tatrensis stadleri). Ces données sont partiellement récapitulées dans l'ouvrage de Schellenberg, 1942. Néanmoins, il ne nous semble pas superflu de donner une nouvelle description complète, et surtout de nouvelles illustrations, de $N$. puteanus, en prenant comme point de départ les normes établies dans la publication de Gibert et al. (1972).

\section{LE MATERIEL ÉTUDIE}

Lors d'une courte excursion d'échantillonnage en août 1973, l'auteur, accompagné de Mlle. A. Goedmakers et de M. le docteur S. Pinkster, a visité une quinzaine de stations autour de la sta-

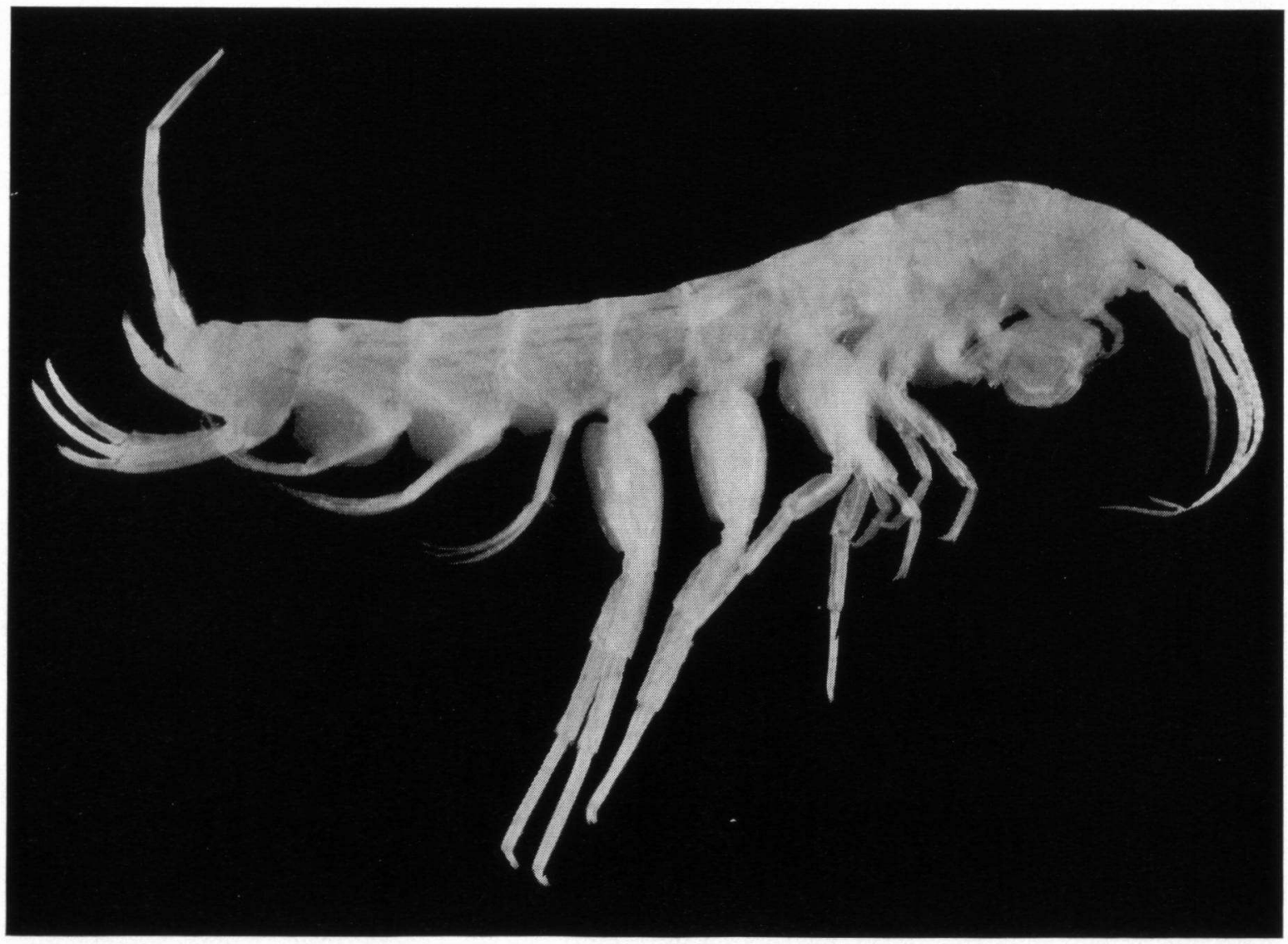

Planche I. L'habitus d'un mâle de Niphargus puteanus (Koch in Panzer, 1836). Longueur actuelle: 13 mm. 
tion-type, à Ratisbonne. On a rencontré des Niphargiens dans cinq de ces stations; tout le matériel récolté se révéla appartenir à une seule espèce: $N$. puteanus. En outre, grâce à l'aimable collaboration du docteur H.-E. Gruner, conservateur du Musée de Zoologie de l'Université Humboldt (Berlin, République Démocratique d'Allemagne), on put étudier le matériel sur lequel Schellenberg (1933a, 1933b) basait ses notes. Ce matériel provient également des environs de Ratisbonne. Le matériel original de Koch étant introuvable (voir Goedmakers, 1972), je juge utile, pour la précision de la nomenclature, d'introduire un néo-type pour $N$. puteanus. Or, Koch ne précise pas exactement où il a trouvé son matériel, il ne dit que „près de Ratisbonne". De la description de Gammarus fossarum (voir Koch in Panzer, 1836, no. 138.1), il apparaît que Koch, aux environs de Ratisbonne, a en tout cas visité la station de „Weichselmühle”.

Il m'a donc paru assez logique d'opter pour la Weichselmühle comme station-type délimitée; de cette station, un bel échantillon de $N$. puteanus a été prélevé pendant l'échantillonnage d'août 1973, dans un puits alimenté par une source.

Les échantillons suivants ont été étudiés:

1. et 2. Deux échantillons ont été récoltés par „Herrn Reichsbahningenieur" (M. l'ingénieur des Chemins de Fer nationaux allemands) Sälzl, dans une source de Ziegetsdorf, faubourg situé au sud de Ratisbonne (Musée de Zoologie de Berlin, cat. no. 23767).

3. Weichselmühle (s'écrit aussi Weichslmühle) près du village de Pentling, arrondissement de Ratisbonne; échantillons prélevés dans un puits maçonné, alimenté par une source; 29 août 1973. De ces échantillons, un mâle adulte a été sélectionné et désigné comme néotype. (Musée de Zoologie d'Amsterdam, no. Amph. 103.912).

4. Même station que 3 , dans la petite source à ciel ouvert d'un ruisseau; 29 août 1973. (Musée de Zoologie d'Amsterdam, no. Amph. 103.914).

5. Dans une petite source de faible débit, jaillissant du sol, à $1,5 \mathrm{~km}$ au SSO de Grasz, arrondissement de Ratisbonne; fond argileux; 28 août 1973. (Musée de Zoologie d'Amsterdam, no. Amph. 103.911).

6. Source latérale du ruisseau Iradingsbach, au N-E de Graszlfing, arrondissement de Ratisbonne; fond de gravier; 28 août 1973. (Musée de Zoologie d'Amsterdam, no. Amph. 103.915).

7. Eau filtrant du sol, au sud de Graszlfing, à côté de la Route 16; entre des joncs et des menthes; 28 août 1973. (Musée de Zoologie d'Amsterdam, no. Amph. 103.913).

\section{DESCRIPTION}

Mâle (Pl. I; figs. 1-4, 5h): Longueur, mesurée du bord frontal de la tête à la base de l'abdomen:
12 à $13 \mathrm{~mm}$. Les descriptions et illustrations ont été faites d'après un exemplaire de cette taille, correspondant à la longueur trouvée par Koch en 1836. Un des échantillons de Ziegetsdorf présente quelques mâles d'une longueur de $20 \mathrm{~mm}$. Je n'ai pas vu d'individus de $30 \mathrm{~mm}$, comme ceux signalés par Schellenberg en 1942.

- Première antenne. Les articles 1,2 et 3 du pédoncule mesurent respectivement $1,00,0,75$ et $0,35 \mathrm{~mm}$. Ils ne portent que de courtes soies. Le long flagelle principal comprend 23 articles. Courts bâtonnets hyalins (environ un tiers de la longueur des articles flagellaires) présents. Le flagelle porte de part et d'autre 2 à 4 soies très courtes. Flagelle accessoire à 2 articles.

- Seconde antenne. L'article 4 porte 3 groupes latéraux et 4 groupes ventraux de soies; les plus longues de celles-ci sont un peu plus longues que le diamètre du segment où elles sont implantées. L'article 5 porte 3 groupes ventraux et 2 groupes latéraux de soies, de même longueur que sur le segment 4 . Flagelle 12-articulé; soies à peu près aussi longues que le diamètre des articles flagellaires.

- Pièces buccales. Pars molaris des mandibules gauche et droite quelque peu asymétriques. Palpe mandibulaire à 3 articles; l'article 3 porte 1 ou 2 groupes de soies ,sur le plat" (= sur les surfaces médiane et latérale), chaque groupe comprenant de 4 à 7 éléments; le bord ventral porte une rangée de soies, quelque peu irrégulière et pectiniforme; 2 soies plus longues, implantées en position subterminale, et 6 soies en position terminale; on rencontre en outre un champ de rugosités le long de la face ventrale.

- Première maxille à lobe interne digitiforme pourvu de 6 soies terminales; lobe externe pourvu de 7 éléments terminaux en forme d'épines; l'élément intérieur porte 3 denticules médians, les autres éléments n'ont qu'un seul denticule médian.

- Seconde maxille: lobe externe pourvu d'environ 22 soies distales, lobe interne de 4 soies médianes et de 26 soies terminales.

- Lèvre inférieure: présence de lobes internes.

- Maxillipède: lobe interne court et distalement pourvu de 5 épines et de 5 soies; lobe externe pourvu d'une rangée médiane de 9 épines et d'une rangée distale d'une dizaine de soies. L'article 1 du palpe va à peu près aussi loin que le lobe interne.

- Gnathopode 1: plaque coxale à peu près $11 / 3 \times$ plus longue que large, bord inférieur pourvu 

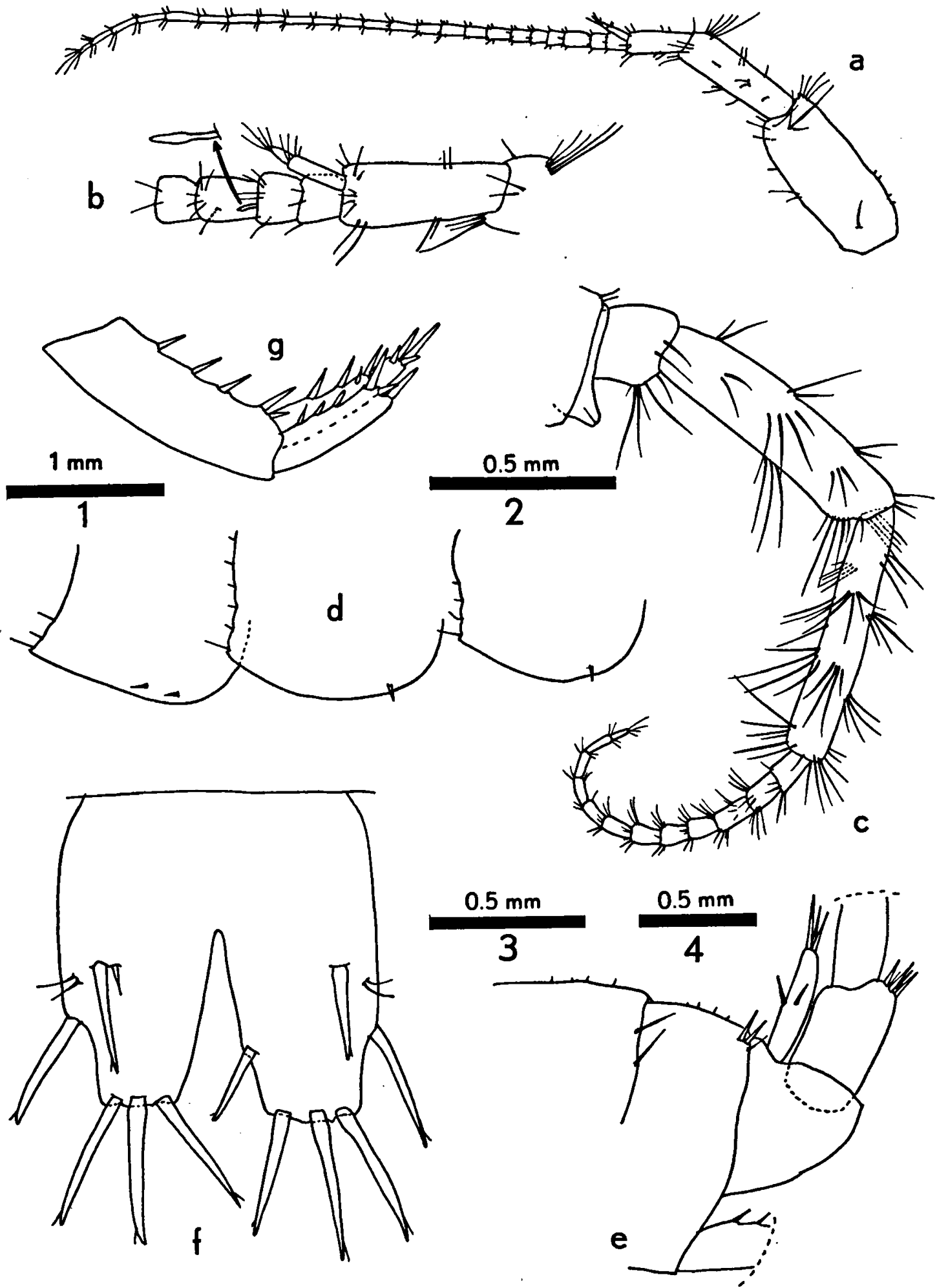

Fig. 1. Niphargus puteanus (Koch in Panzer, 1836), $\delta$. a, antenne 1 (échelle 1); b, partie de l'antenne 1 portant le flagelle accessoire (éch. 2); c, antenne 2 (éch. 3); d, plaques épimérales 1 (à droite) à 3 (à gauche) (éch. 1); e, urosome, de gauche (éch. 4); f, telson, dorsal (éch. 5); g, uropode 2 (éch. 4). 


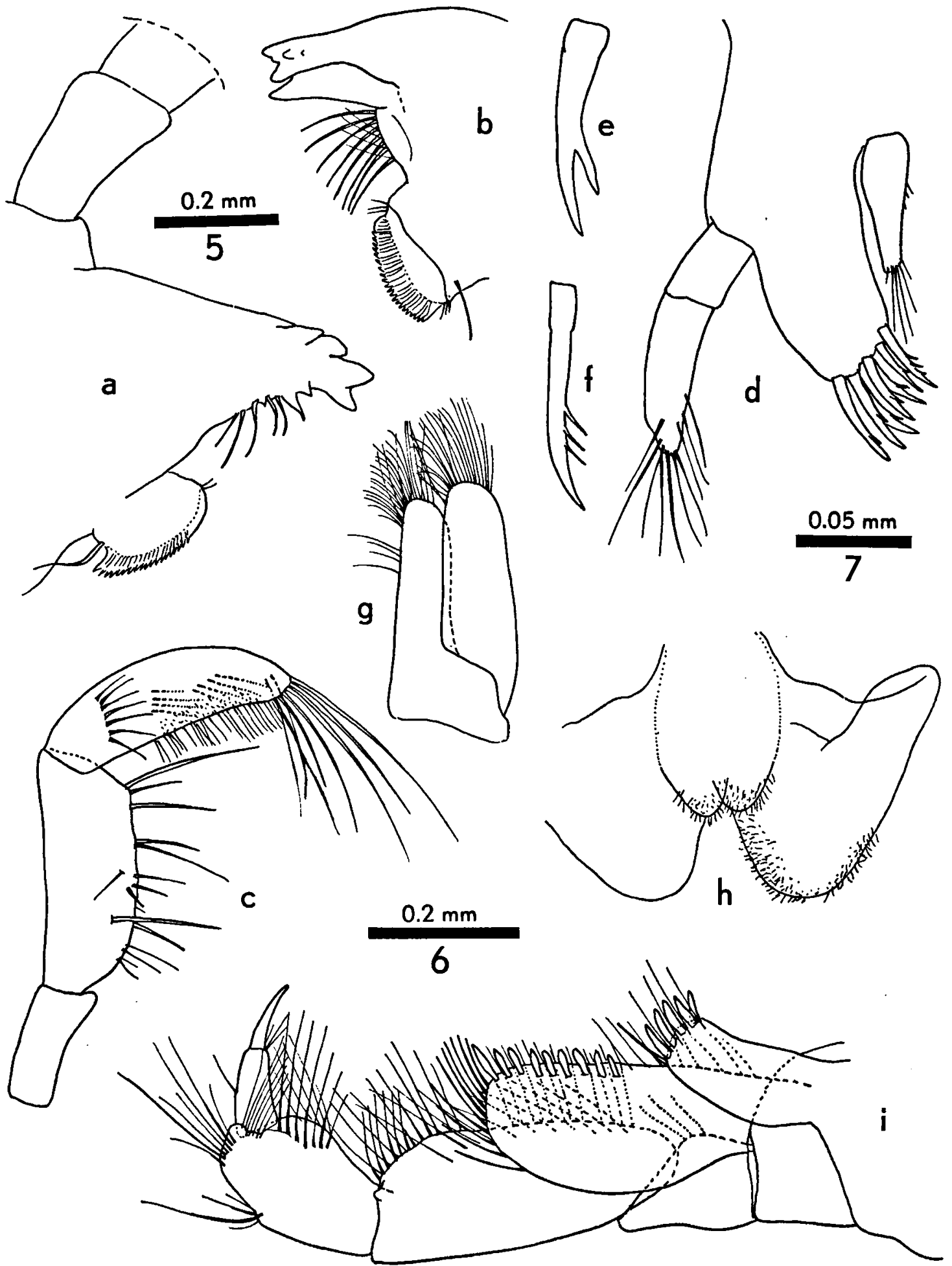

Fig. 2. Niphargus puteanus (Koch in Panzer, 1836), §. a, b, pars molaris des mandibules contralatérales (échelle 6); c, palpe mandibulaire (éch. 5); d, maxille 1 (éch. 6); e, ant épénultième dent distale du lobe extérieur de la maxille 1 (éch. 7); f, dent distale la plus médiane du lobe extérieur de la maxille 1 (éch. 7); g, maxille 2 (éch. 5); h, lèvre inférieure (éch. 5); $i$, maxillipède (éch. 5). 

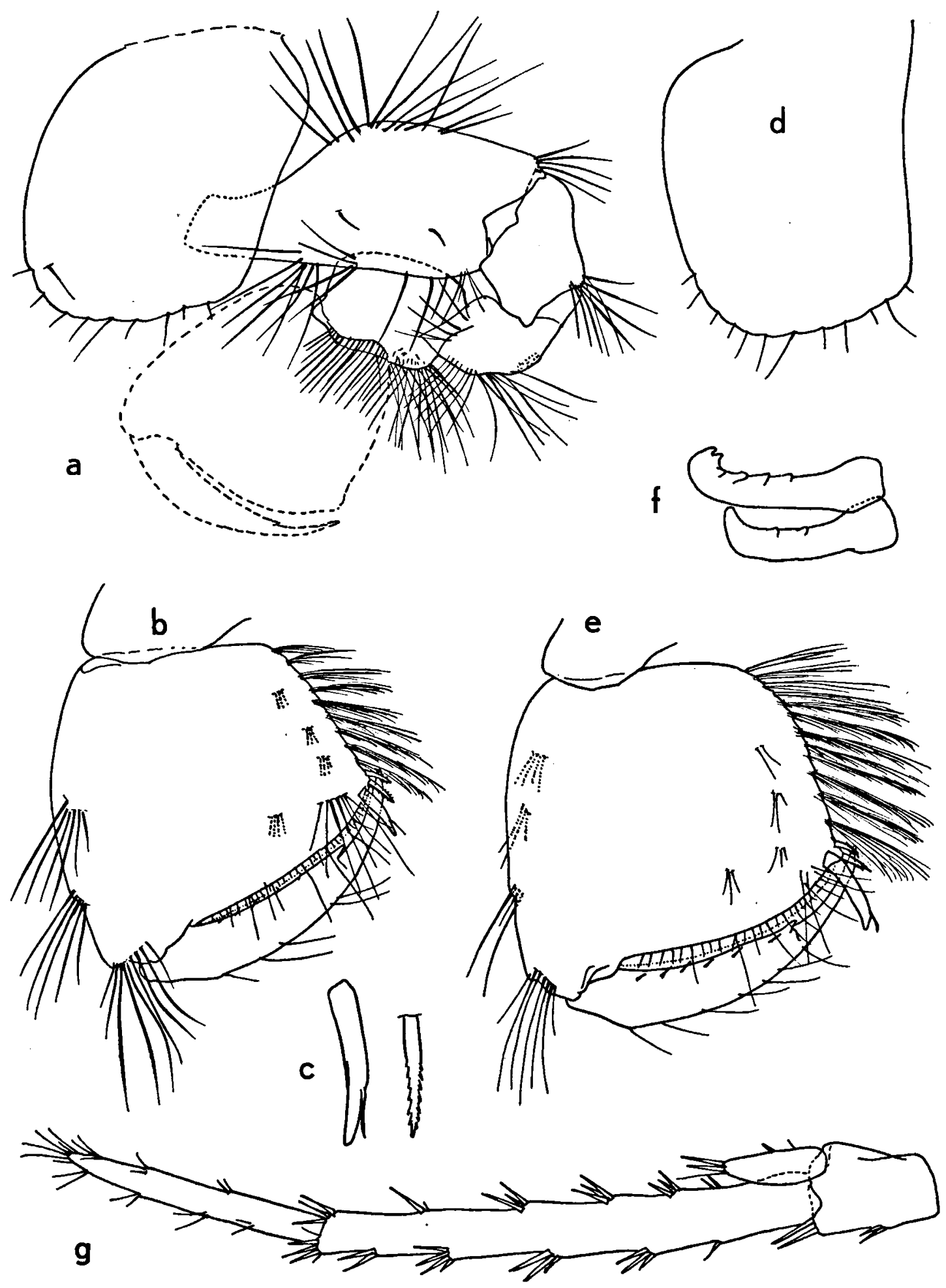

Fig. 3. Niphargus puteanus (Koch in Panzer, 1836), $\hat{\delta}$. a, gnathopode 1 (echelle 3); b, propode du gnathopode 1 (éch. 2); c, épines de l'angle palmaire du gnathopode 1 (croquis à main levée); d, plaque coxale 2 (éch. 3); e, propode du gnathopode 2 (éch. 2); f, rétinacles du pléopode 3 (croquis à main levée); g, uropode 3 (éch. 4). 


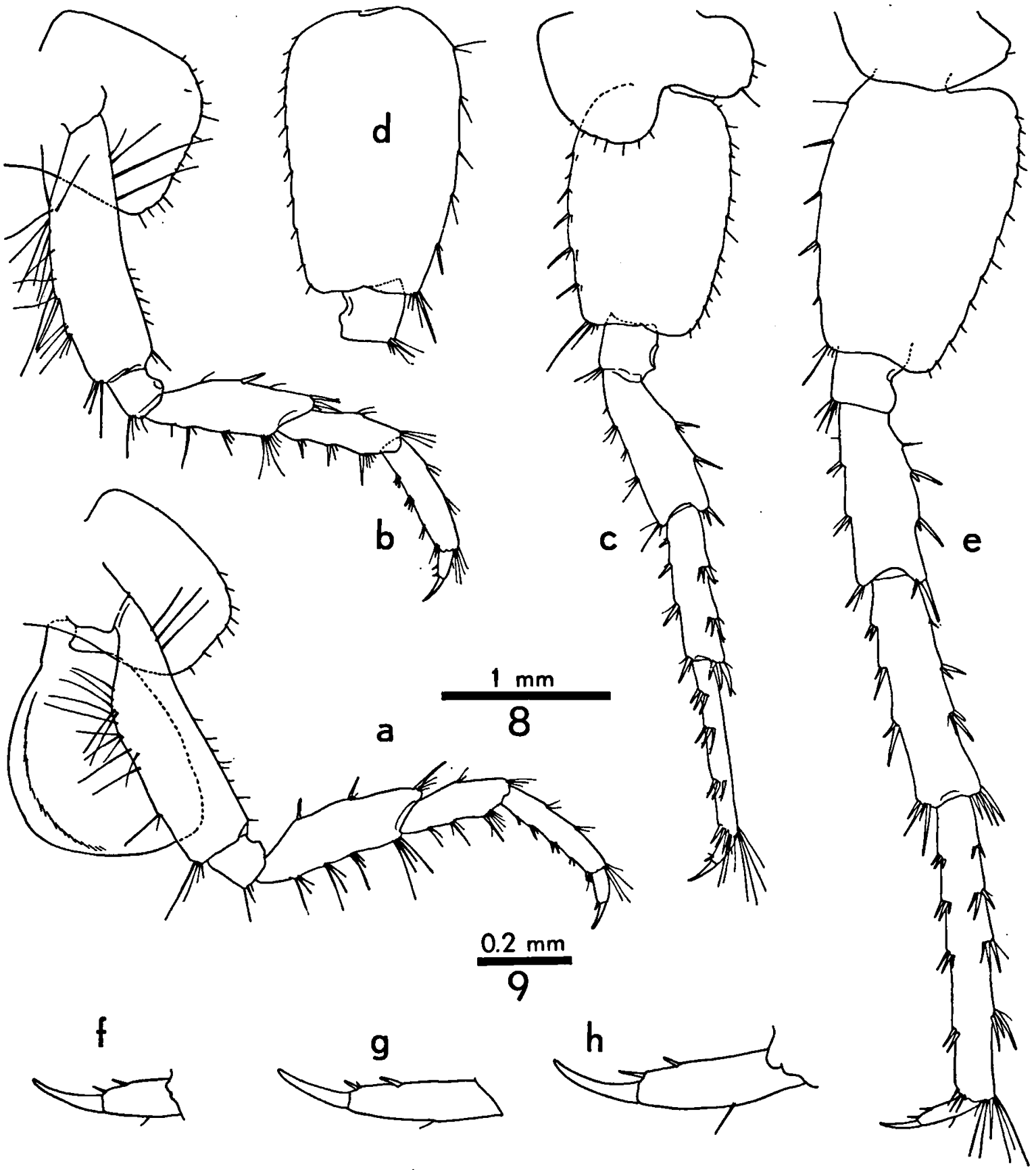

Fig. 4. Niphargus puteanus (Koch in Panzer, 1836), ô. a, péréiopode 3 (échelle 8); b, péréiopode 4 (éch. 8); c, péréiopode 5 (éch. 8); d, basis du péréiopode 6 (éch. 8); e, péréiopode 7 (éch. 8); f, griffe du péréiopode 4 (éch. 9 ); g, griffe du péréiopode 6 (éch. 9); h, griffe du péréiopode 7 (éch. 9).

d'une rangée de soies courtes. Le propode est légèrement plus large que long, rectangulaire à trapézoïdal. Angle palmaire armé de 2 épines courtes dont les bords latéraux sont sciés, et d'une épine plus longue ciliée latéralement dans la partie distale. Bord interne du dactyle pour- 

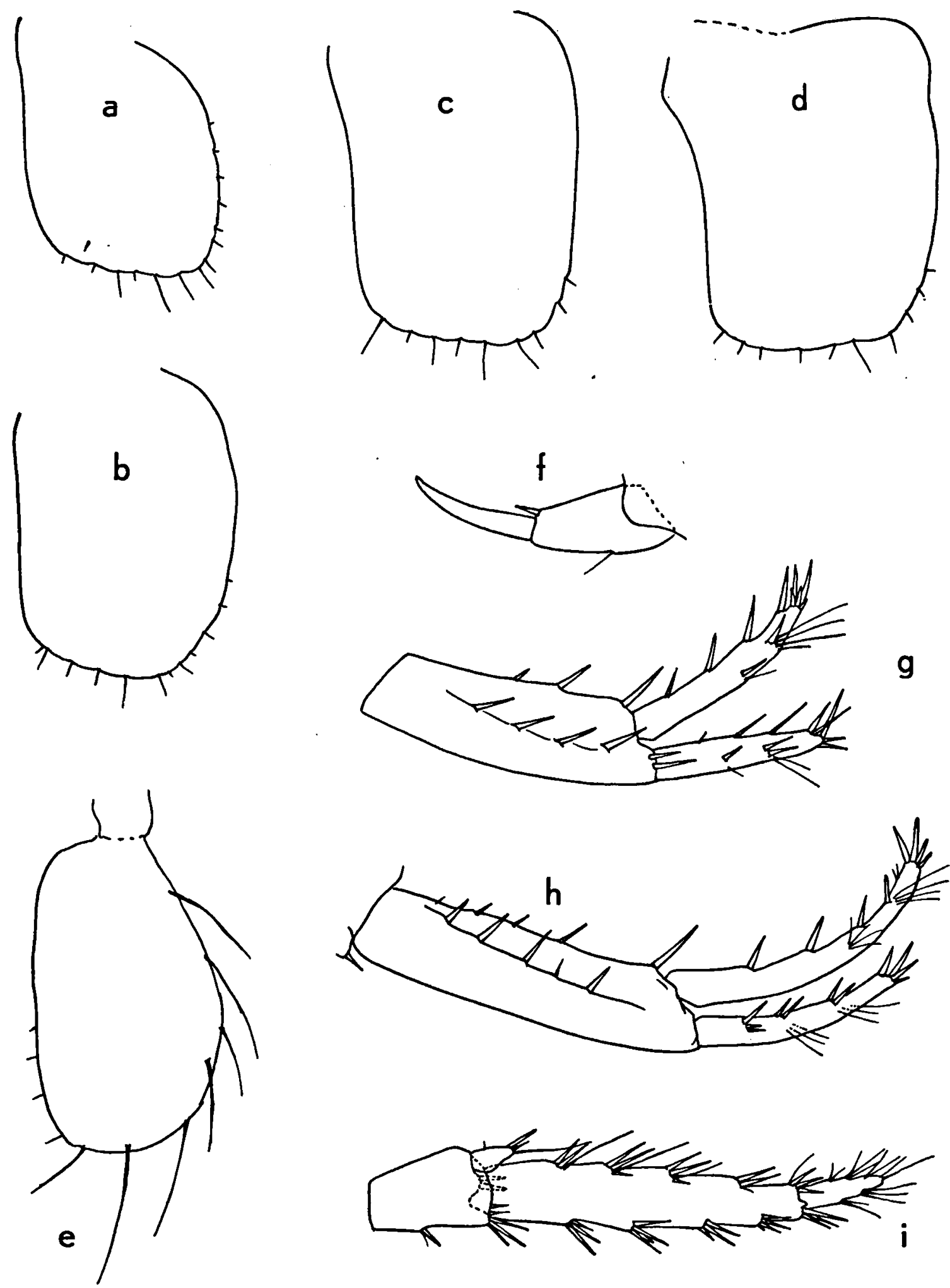

Fig. 5. Niphargus puteanus (Koch in Panzer, 1836). a, plaque coxale 1, \& (échelle 3); b, plaque coxale 2, $q$ (éch. 3); c, plaque coxale 3, \& (éch. 3); d, plaque coxale 4, \& (éch. 3); e, oostégite, $\&$ (éch. 3); f, griffe du péréiopode 4, $q$ (éch. 6); g, uropode 1, \& (éch. 4); h, uropode 1, ô (éch. 4); i, uropode 3, \& (éch. 4). 
vu d'un processus triangulaire et, en position proximale de celui-ci, de quelques petits cils très courts. Le bord externe du dactyle est pourvu de 5 soies longues.

- Gnathopode 2: plaque coxale plus longue que large, bord inférieur pourvu de soies courtes. Propode presque rectangulaire, légèrement plus large que long. Epines angulaires comme dans le gnathopode 1, l'épine majeure étant toutefois très fortement développée. Armature du dactyle comme celle du gnathopode 1 .

- Péréiopode 3: plaque coxale légèrement plus longue que large. Bord interne du dactyle muni d'une petite épine.

- Péréiopode 4: plaque coxale rectangulaire, presque aussi longue que large. Bord interne du dactyle muni de 2 petites épines.

- Péréiopode 5: hauteur maximale de la plaque coxale environ les $4 / 5$ de celle de la quatrième patte. Base rectangulaire allongée, bord antérieur muni de petites épines, bord postérieur cilié (une quinzaine de cils). Dactyle pourvu parfois d'une épine, parfois de deux.

- Péréiopodes 6 et 7 pratiquement semblables l'un à l'autre. Base rectangulaire allongée. Dactyle muni de 2 épines.

- Branchies larges et ovales, sans particularités.

- Plaques épimérales: bord inférieur muni d' 1 ou 2 épines; bord postérieur cilié; la première et la deuxième plaque ont une face postérieure rectangulaire, la troisième plaque a cette face légèrement pointue.

- Pléopodes: les branches sont constituées d'une quinzaine d'articles. Le rapport entre la base et la longueur des brances est à peu près de 100 à 130. Chaque pléopode porte 2 rétinacles en forme de crochet, munis de 2 à 5 denticules obtus, répartis sur leur bord interne.

- Le premier segment de l'urosome a 2 soies dorso-latérales; le deuxième segment a 2 épines et 1 cil de part et d'autre; le troisième segment n'est pas armé.

- Telson: la fente médiane va jusqu'aux $3 / 5$ de la longueur totale. L'armature est variable; en général, il y a 3 épines distales, 1 épine latérale, 1 ou 2 épines dorsales et 2 soies sensorielles. Les moitiés contralatérales du telson sont souvent différemment armées.

- Uropode 1: basipodite à deux rangées de 5 épines chacune. Branche interne certainement $1 / 4$ plus longue que la branche externe; les deux branches sont armées d'épines et pourvues de quelques soies.
- Uropode 2: les deux branches ne sont armées que d'épines. La branche interne est un peu plus longue que la branche externe.

- Uropode 3: très allongé. L'article proximal de la branche externe est presque uniquement armé de petites épines. L'article distal n'est que cilié. Branche interne digitiforme, environ 5 fois plus courte que le premier article de la branche externe.

Femelle (figs. 5a-g, i): les plaques coxales 1 à 4 inclus sont un peu plus allongées que chez le mâle. Les oostégites sont à peu près ovales toutes, sauf celles du gnathopode 2 , à longues soies. Le bord interne du dactyle de la patte 4 est unidenticulé. La branche interne de l'uropode 1 est peu allongée (cette branche est environ $15 \%$ plus longue que la branche externe). L'uropode 3 est moins fortement allongé, l'article distal de la branche externe est court; la branche interne est courte. Une femelle portant 16 oeufs dans le marsupium avait une longueur de $11 \mathrm{~mm}$; le diamètre des oeufs atteignait environ $810 \times 630$ microns. Les autres caractéristiques correspondent à celles du mâle.

Remarques: En attendant la nouvelle description de quelques autres espèces „classiques” du genre Niphargus, telles que longicaudatus et stygius, il est prématuré de discuter ici les parentés de cette espèce. Remarquons cependant que dans de nombreuses descriptions plus anciennes, surtout d'avant 1933, lorsque Schellenberg retrouva l'espèce près de la station-type, le nom de $N$. puteanus est employé à tort. Les synonymes les plus connus sont les suivants:

Niphargus puteanus sensu S. Karaman, 1932: 180 , est en réalité $N$. aquilex.

Niphargus puteanus sensu Schellenberg, 1932: 321 , est en réalité $N$. fontanus.

Niphargus stadleri G. Karaman, 1932: 185 (N. tatrensis stadleri de Schellenberg, 1932: 316) correspond à l'authentique $N$. puteanus.

Niphargus puteanus de Chevreux \& Fage, 1925, est en réalité $N$. schellenbergi.

\section{REFERENCES BIBLIOGRAPHIQUES}

Chevreux, Ed. \& L. Fage, 1925. Amphipodes. Faune de France, $9: 1-488$.

Gibert, J., R. Ginet \& C. Morand-Chevat, 1972. Normes pour la description d'unités taxonomiques du genre Niphargus (Crustacé Amphipode hypogé). Memorie fuori Ser. Mus. civ. Storia nat. Verona, 5 : $65-70$.

GiNET, R., sous presse a. Le deuxième colloque inter- 
national sur les genres Niphargus et Gammarus Lyon, 1973. Ann. Spéléol.

-, sous presse b. Le deuxième colloque international sur les genres Niphargus et Gammarus - Lyon, 1973. Intern. J. Speleol.

GoEDMAKERS, A., 1972. Gammarus fossarum Koch, 1835: redescription based on neotype material and notes on its local variation (Crustacea, Amphipoda). Bijdr. Dierk., 42 (2) : 124-138.

KarAman, S., 1932. Beitrag zur Kenntniss der SüsswasserAmphipoden, 5. Amphipoden unterirdischer Gewässer. Prirodosl. Razpr., 2 : $179-232$.

Koch, C. L., 1837. Deutschlands Crustaceen, Myriapoden und Arachniden, Heft 5. (G. A. W. Herrich-Schäffer, Regensburg).

一, 1844. Ibid., Heft 36.

PANZER, G. W. F. (ed.), 1836. Faunae Insectorum Germanicae initia, Heft 138. (Felsecker, Nürnberg).
一, 1844. Ibid., Heft 186.

RUFFo, S., 1972. I. Colloquio internazionale sul genere Niphargus. Memorie fuori Ser. Mus. civ. Storia nat. Verona, 5 : vii-ix.

SChEllenBERG, A., 1932. Deutsche subterrane Amphipoden. Zool. Anz., 99 (11-12) : 311-323.

-, 1933a. Niphargus puteanus am alten Fundort neu entdeckt. Revision der Deutschen Niphargusarten. Zool. Anz., $102(9-10)$ : 255-257.

,$- 1933 b$. Niphargus-Probleme. Mitt. zool. Mus. Berl., 19 : $406-429$.

-, 1942. Flohkrebse oder Amphipoda. Tierwelt Dtl., 40 (4) : i-iv, 1-252.

SHerborN, C. D., 1923. On the dates of C. L. Koch, 'Deutschlands Crustaceen, Myriapoden und Arachniden', 1835-44. Ann. Mag. nat. Hist., (9) 11 : 568.

WRZESNIOWSKI, A., 1890. Ueber drei unterirdische Gammariden. Z. wiss. Zool., $50: 600-724$.

Reçu : le 15 octobre 1973 